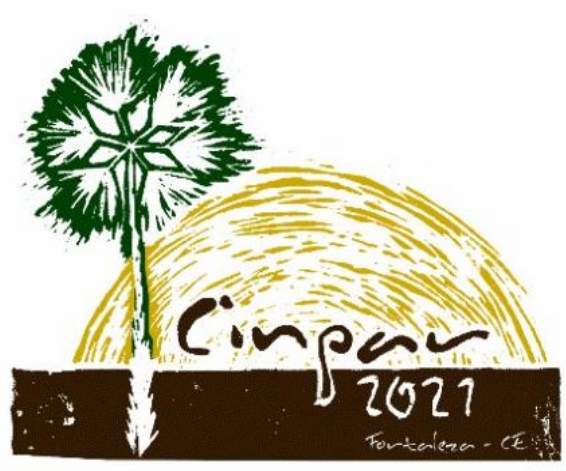

XVII Congresso Internacional sobre Patologia e

Reabilitação das Construções

XVII Congreso Internacional sobre Patología y Rehabilitación de las Construcciones

XVII International Conference on Pathology and Constructions Rehabilitation

FORTALEZA (Brasil), 3 a 5 de junho de 2021

https://doi.org/10.4322/CINPAR.2021.029

\title{
Durabilidade do UHPC e UHPFRC contendo metacaulim
}

\section{Durability of the UHPC and UHPFRC containing metakaolin}

\author{
Luis Henrique Pereira França ${ }^{1}$, Marcelo Silva Medeiros Júnior ${ }^{2}$, Alexandre de Miranda Mont'alverne \\ ${ }^{1}$ Universidade Federal do Ceará, Fortaleza, Ceará, luishenrique1924@hotmail.com \\ ${ }^{2}$ Universidade Federal do Ceará, Fortaleza, Ceará, marcelo.medeiros@ufc.br \\ ${ }^{3}$ Universidade Federal do Ceará, Fortaleza, Ceará, montalverne@ufc.br
}

\begin{abstract}
Resumo: O Ultra High Performance Concrete (UHPC) apresenta elevada resistência à compressão, tração, durabilidade, elevado módulo de elasticidade, baixa porosidade e permeabilidade. As estruturas de UHPC são mais leves quando comparadas com estruturas de concreto armado e protendido. Embora o custo inicial do UHPC seja elevado devido o alto consumo de cimento e fibras metálicas, a adição de materiais cimentícios secundários tende a diminuir o consumo de cimento e é uma opção sustentável. O UHPC é amplamente utilizado na Europa, Canadá e China em estruturas como pontes, passarelas, aranha-céus e reabilitação estrutural. Por ter uma microestrutura densa, o UHPC apresenta bom comportamento frente a penetração da água e agentes agressivos ao concreto e a armadura passiva. Nesse sentido, no presente trabalho foi analisado a absorção por capilaridade em corpos de prova cilíndricos de $5 \mathrm{~cm}$ de diâmetro e $10 \mathrm{~cm}$ de altura. Foi estudado a capilaridade no UHPC e no Ultra High Performance Fiber Reinforced Concrete (UHPPFC), sendo observado que a ordem dos materiais adicionados na argamassadeira afeta a trabalhabilidade e a resistência à compressão. Além disso, a adição de fibras pouco influenciou na absorção por capilaridade e a variação da massa permaneceu, quase que constante, no UHPC e UHPFRC. Os resultados obtidos já eram esperados, pois a microestrutura do UHPC possui poucos vazios, desse modo é praticamente impermeável.
\end{abstract}

Palavras-chave: UHPC. UHPFRC. Durabilidade. Capilaridade. Fibras metálicas.

Abstract: Ultra High Performance Concrete (UHPC) has high resistance to compression, tensile, durability, high modulus of elasticity, low porosity and permeability. UHPC structures are lighter when compared to reinforced and prestressed concrete structures. Although the initial cost of UHPC is high due to the high consumption of cement and steel fibers, the addition of secondary cementitious materials tends to decrease the consumption of cement and is a sustainable option. UHPC is widely used in Europe, Canada and China in structures such as bridges, walkways, skyscrapers and structural rehabilitation. As it has a dense microstructure, the UHPC has a good behavior against water penetration and aggressive agents to concrete and passive reinforcement. In this sense, in the present work, the absorption by capillarity in cylindrical specimens of $5 \mathrm{~cm}$ in diameter and $10 \mathrm{~cm}$ in height was analyzed. Capillarity in UHPC and Ultra High Performance Fiber Reinforced Concrete (UHPPFC) was studied, and it was observed that an order of materials created in the mortar affects workability and resistance to compression. In addition, the addition of fibers has little influence on capillarity absorption and mass variation remained almost constant in UHPC and UHPFRC. The results obtained were already expected, as the microstructure of the UHPC has few voids, so it is practically impermeable.

Keywords: UHPC. UHPFRC. Durability. Capillarity. Steel fibers. 


\section{Introdução}

A inovação da construção civil se da por meio do desenvolvimento de novos materiais e novas tecnologias, com a finalidade de tornar as estruturas mais leves e duráveis. O Ultra High Performance Concrete (UHPC) possui resistência à compressão superior a $150 \mathrm{MPa}$ e uma microestrutura muito densa, quase que impermeável (XU et al., 2019). A união de bom desempenho mecânico e microestrutura altamente densa favorece a utilização desse tipo de concreto em estruturas, em estruturas especiais, em ambientes com alta agressividade ambiental e como reforço de estruturas deterioradas (FEHLING et al., 2014).

A durabilidade do UHPC é afetada, por exemplo, pela adição de materiais cimentícios secundários (SCM) como a sílica, metacaulim e quartzo que tendem a diminuir a retração autógena e garantir que os vazios existentes entre os grãos de areia e cimento sejam preenchidos por materiais com granulometria inferior (CAREY et al., 2020). Com isso, confere ao UHPC uma microestrutura impermeável, dificultando a percolação da água, agentes químicos e biológicos. Em consonância, Mehta e Monteiro (2017) afirmam que a permeabilidade é um fator primário na definição de durabilidade e que materiais apresentam baixa permeabilidade e porosidade apresentará elevada durabilidade.

Segundo a NBR 6118 (2014), a durabilidade está correlacionada com as intervenções ao longo da vida útil da estrutura, desse modo, uma elevada durabilidade está interligada com a preservação das características de projeto e vida útil. O UHPPFC é a evolução do UHPC, apresentando maior ductilidade e melhor desempenho mecânico, devido a adição de fibras metálicas, na análise das propriedades de resistência à compressão, tração, energia de fratura e tenacidade. O UHPC apresenta maior durabilidade e leveza, quando se considera a mesma capacidade portante em diferentes tipos de estruturas. Uma estrutura de UHPC é três vezes mais leve que o concreto protendido e quatro vezes mais leve que o concreto armado com armadura passiva. Logo, é uma opção sustentável para a construção civil. Como pode ser visto na Figura 1, o UHPC reduz as seções transversais (CHRIST et al., 2019; FANG et al., 2020).

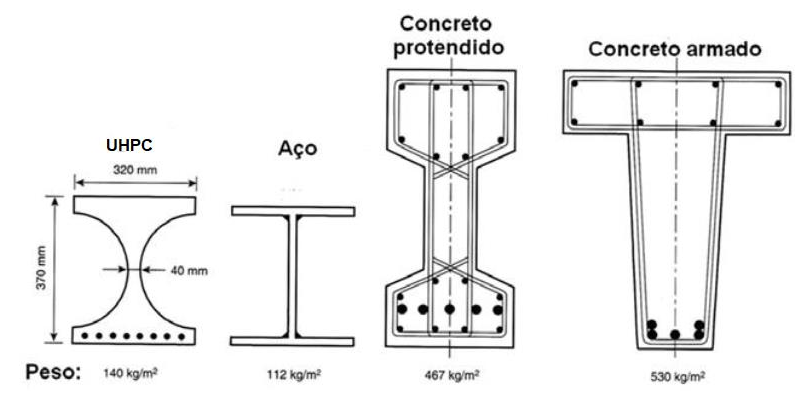

Figura 1 - Peso próprio e capacidade portante das estruturas, adaptado de Walraven (2000).

Como mostra a Figura 2, o UHPC pode ser utilizado em ambientes que exigem elevada resistência à corrosão, baixa permeabilidade e porosidade. Por possuir um ciclo de vida superior aos demais sistemas da Figura 1, as intervenções ao longo do tempo são menores, não necessitando de reforços e reparos periodicamente, logo é financeiramente vantajoso quando se analisa o ciclo de vida do UHPC (DAIRON, 2016). Segundo Bae e Pyo (2020) e Alsalman et al. (2020), as fibras metálicas devem ser adicionadas para aumentar a durabilidade e diminuir as fissuras de tração, pois o UHPC apresenta uma falha quebradiça e frágil. Desse modo, pode facilitar a percolação de água e agentes nocivos ao concreto e à armadura.

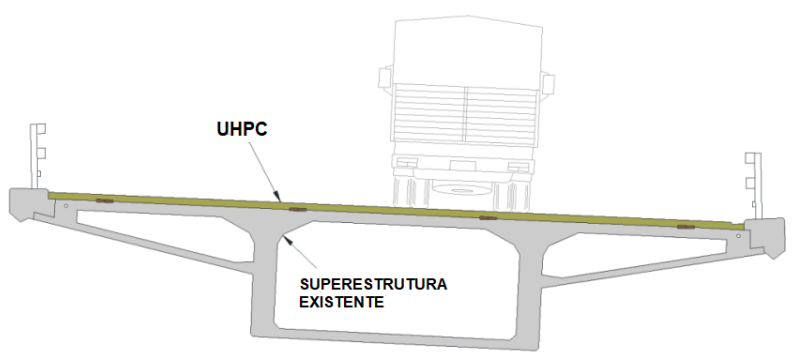

Figura 2 - Uso do UHPC como reforço em pontes, adaptado de Haber et al. (2018). 
No presente estudo foi analisado a capacidade de absorção por capilidaridade do UHPC com e sem fibras. Uma vez que a percolação de agentes químicos e biológicos é baixa, o material desenvolvido pode ser utilizado em elementos estruturais que demandam uma rápida intervenção e necessidade de devolver a capacidade portante da estrutura. Por consequência, pode ser utilizado em obras especiais como, por exemplo, estruturas dispostas em ambientes com classe de agressividade ambiental elevada como respingo de maré, industrial e offshore.

\section{Metodologia}

O presente trabalho foi subdivido em duas etapas. Na primeira etapa foi realizado um estudo do traço por meio do empacotamento de partículas, depois o procedimento de mistura, em seguida a definição do tipo de cura, para finalmente ser realizado o ensaio de compressão axial. Na segunda etapa foi realizado o ensaio de absorção por capilaridade no UHPC e UHPFRC.

\subsection{Traço, mistura, cura e ensaio de compressão axial}

Por meio de um longo estudo do procedimento de mistura e de escolha dos materiais para atingir uma resistência à compressão superior a $150 \mathrm{MPa}$ em cura padrão chegou-se no traço disposto no Quadro 1 .

Quadro 1 - Traço desenvolvido

\begin{tabular}{|c|c|c|c|c|}
\hline Cimento & Areia & Metacaulim & Água & Superplastificante \\
\hline 1 & 1,02 & 0,25 & 0,20 & 0,04 \\
\hline
\end{tabular}

O traço foi definido por meio do empacotamento de partículas procurou-se otimizar a mistura retirando o agregado graúdo e adicionando materiais cimentícios secundários. O empacotamento de partículas foi realizado por meio da Eq.1, nesse sentido duas ferramentas computacionais foram utilizadas, o software EMMA e o Solver do Excel.. Essa metodologia é conhecida como A\&A modificado e foi proposto por Funk e Dinger (1994).

$$
\frac{C P F T}{100 \%}=\frac{D^{q}-D_{\min }^{q}}{D_{\max }^{q}-D_{\min }^{q}}
$$

Onde:

- $D_{\max }$ é o maior diâmetro; $D_{\min }$ é o menor diâmetro; Porcentagem acumulada passante de finos.

A fibra metálica utilizada foi do tipo Dramix 3D 45/50 BL, com fator de forma (I/d) na ordem de 45 e ganchos nas suas extremidades. Com comprimento de $50 \mathrm{~mm}$ e diâmetro de $1,05 \mathrm{~mm}$. A areia utilizada possuía diâmetro máximo característico (DMC) de $2,36 \mathrm{~mm}$. O cimento utilizado foi do tipo CP V -ARI com massa específica de $3,08 \mathrm{~g} / \mathrm{cm}^{3}$. O metacaulim HP Ultra foi utilizado para preencher os vazios entre os grãos de areia e cimento, tendo massa específica igual a $2,56 \mathrm{~g} / \mathrm{cm}^{3}$. O superplastificante (SP) Power Flow 4001 foi uitlizado nessa pesquisa. $\mathrm{O}$ ensaio de saturação do SP foi realizado para verificar a compatibilidade com o cimento. $\mathrm{A}$ água utilizada na mistura foi da rede de abastecimento público da cidade de Fortaleza - CE.

A mistura dos materiais foi realizada por meio de uma argamassadeira de $6 \mathrm{~L}$. Foram moldados corpos de prova $(C P)$ no formato cúbico $(4 \times 4 \times 4 \mathrm{~cm})$ e prismático $(4 \times 4 \times 16 \mathrm{~cm})$ para determinação da resistência à compressão. Os corpos de prova cilíndricos de $5 \mathrm{~cm}$ de diâmetro e $10 \mathrm{~cm}$ de altura foram moldados para realização do ensaio de absorção por capilaridade. Para a resistência à compressão foram moldados 3 CPs para cada idade, para o ensaio de capilaridade foram moldados $3 \mathrm{CPs}$ para a condição com e sem fibras. A cura adotada foi a cura padrão em temperatura ambiente, como indica a NBR 5738 (2015). E por fim, a Figura 3 representa a metodologia da moldagem até o ensaio de compressão axial. 


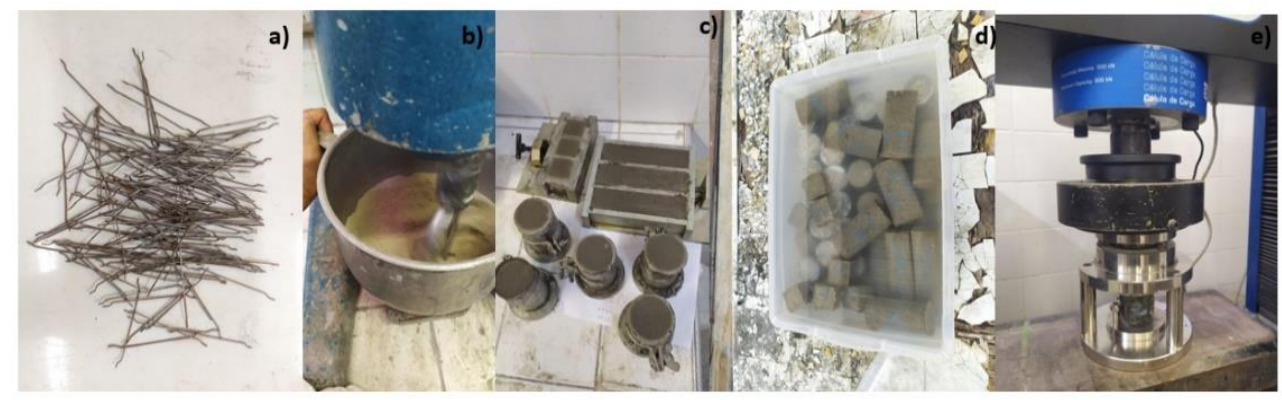

Figura 3 - Fibra, traço, mistura, moldagem, cura e ensaio de compressão axial.

2.2 Ensaio de absorção por capilaridade

Toda a metologia deste ensaio foi realizada conforme a NBR 9779 (2012). Para obtenção da massa seca, os corpos de prova foram colocados em uma estufa a $100^{\circ} \mathrm{C}$ por um período de 24 horas, em seguida foi determinada a massa seca e saturada como mostra a Figura 4. Foi colocada uma lâmina de água e os corpos de prova foram dispostos nessa lâmina, a massa saturada foi determinada em 3,6,24,48 e 72 horas.

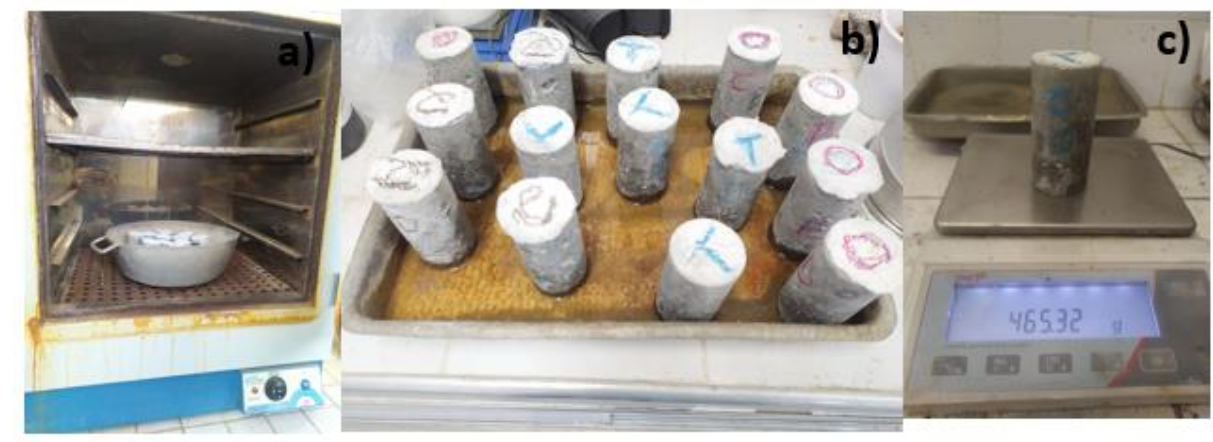

Figura 4 - Ensaio de absorção por capilaridade.

A capilaridade é determinada por meio da Eq. 2.

$$
C=\frac{M_{s a t}-M_{S}}{S}
$$

Onde:

- C é a capilaridade;

- $\mathrm{M}_{\text {sat }}$ é a massa saturada em função do tempo que varia de 3 horas até 72 horas;

- $M_{s}$ é a massa seca após 24 horas na estufa ou até estabilização da massa.

\section{Resultados e discussão}

No presente estudo foram analisadas a resistência à compressão e a absorção por capilaridade. Segundo Alkaysi e El Tawil (2015) e Carey et al. (2020), o UHPC deve apresentar resistência à compressão superior a $150 \mathrm{MPa}$. Tal parâmetro foi alcançado no presente trabalho. No Quadro 2 e 3 são apresentados os resultados obtidos por meio de compressão axial em cura padrão ao 7,14 e 28 dias de idade. Graficamente os resultados podem ser visualizados na Figura 5. 
Quadro 2 - Resistência à compressão do UHPC com 0\% de fibras.

\begin{tabular}{|c|c|c|c|}
\hline \multicolumn{4}{|c|}{ 0\% de fibras (MPa) } \\
\hline CP & 7 dias & 14 dias & 28 dias \\
\hline 1 & 121,19 & 123,62 & 137,79 \\
\hline 2 & 123,90 & 122,69 & 123,56 \\
\hline 3 & 118,40 & 120,31 & 109,52 \\
\hline Média & 121,16 & 122,20 & 123,62 \\
\hline
\end{tabular}

Quadro 3 - Resistência à compressão do UHPFRC com $2 \%$ de fibras.

\begin{tabular}{|c|c|c|c|}
\hline \multicolumn{3}{|c|}{ 2\% de fibras (MPa) } \\
\hline CP & 7 dias & 14 dias & 28 dias \\
\hline 1 & 107,98 & 106,50 & 150,99 \\
\hline 2 & 122,58 & 135,61 & 140,14 \\
\hline 3 & 108,72 & 119,95 & 147,30 \\
\hline Média & 113,09 & 120,68 & 146,14 \\
\hline
\end{tabular}

As fibras foram adicionadas em relação ao volume total de materiais, pois a ruptura do UHPC é extremamente frágil, por isso várias pesquisas adicionam as fibras em relação ao volume e não em relação à massa de cimento (CHRIST et al., 2019; FANG et al., 2020; FEHLING et al., 2014 ). Quanto maior for a resistência à compressão, menor será a ductilidade do concreto, desse modo, as fibras tendem a mitigar essa deficiência tornando o UHPC mais ductil.

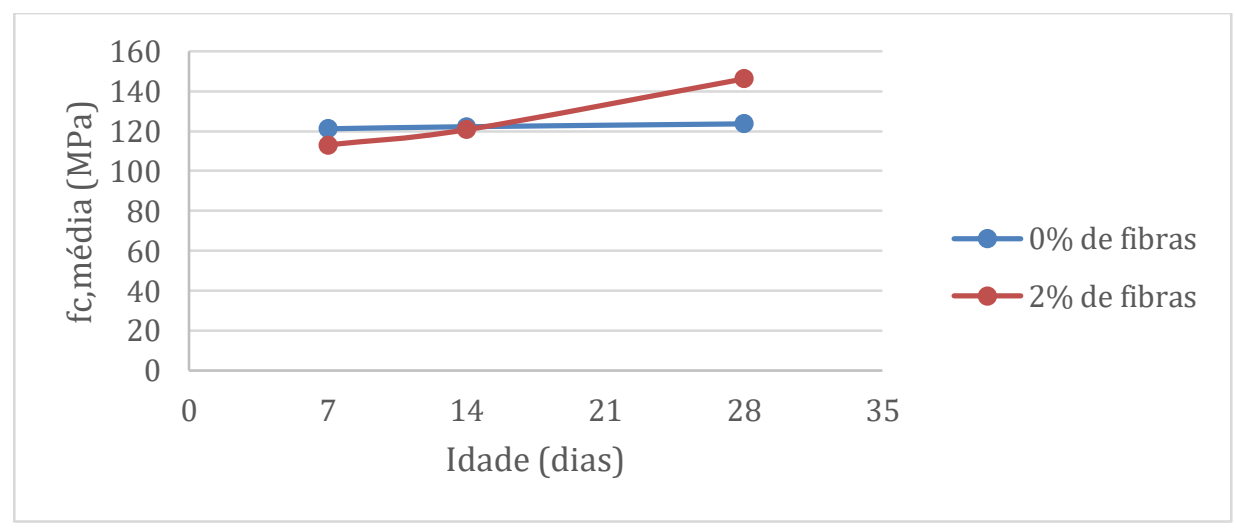

Figura 5 - Resistência à compressão aos 28 dias de idade.

O UHPFRC com $2 \%$ de fibras apresentou uma resistência à compressão $15 \%$ superior ao UHPC sem fibras, aos 28 dias. $\mathrm{O}$ aumento da resistência ocorreu devido a adição de fibras na mistura. Além disso, percebeu-se que o processo de ruptura foi mais frágil no UHPC. Foi notado também que a resistência à compressão do UHPC evoluiu lentamente em função do tempo, isso ocorre pois os materiais cimentícios secundários adicionados (metacaulim) tendem a retardar a hidratação do concreto. Por isso, esse tipo de concreto é testado mecanicamente em idades mais elevadas, tal efeito não foi observado no UHPFRC, pois a adição de fibras melhorou o desempenho mecânico.

Os resultados de absorção por capilaridade estão dispostos nos Quadros 4 e 5. A NBR 9779 (2012) diz que a massa seca deve ser estabilizada onde a diferença entre os CPs não deve ser superior a 0,5\%, entrentanto isso não é possível para o UHPFRC, pois os CPs foram moldados em uma única moldagem, desse modo não é possível garantir que os CPs tivessem a mesma quantidade de fibras. 
Quadro 4 - Média da massa seca e saturada em 3 e 6 horas.

\begin{tabular}{|c|c|c|c|c|c|c|c|c|}
\hline \multicolumn{3}{|c|}{ Massa seca } & \multicolumn{3}{c|}{$\mathbf{M}_{\text {sat }}-\mathbf{3}$ horas } & \multicolumn{3}{c|}{$\mathbf{M}_{\text {sat }}-\mathbf{6}$ horas } \\
\hline CP & $\mathbf{0 \%}$ & $\mathbf{2 \%}$ & CP & $\mathbf{0 \%}$ & $\mathbf{2 \%}$ & CP & $\mathbf{0 \%}$ & $\mathbf{2 \%}$ \\
\hline CP-A & 458,94 & 489,29 & CP-A & 461,33 & 491,87 & CP-A & 461,35 & 491,94 \\
\hline CP-B & 458,31 & 483,94 & CP-B & 460,41 & 485,87 & CP-B & 460,48 & 485,93 \\
\hline CP-C & 459,32 & 493,31 & CP-C & 461,40 & 495,9 & CP-C & 461,51 & 495,81 \\
\hline MÉDIA & 458,85 & 488,84 & MÉDIA & 461,04 & 491,21 & MÉDIA & 461,11 & 491,22 \\
\hline
\end{tabular}

Quadro 5 - Média da massa seca e saturada em 24, 48, 71 horas.

\begin{tabular}{|c|c|c|c|c|c|c|c|c|}
\hline \multicolumn{3}{|c|}{$\mathbf{M}_{\text {sat }}-\mathbf{2 4}$ horas } & \multicolumn{3}{c|}{$\mathbf{M}_{\text {sat }}-\mathbf{4 8}$ horas } & \multicolumn{3}{c|}{$\mathbf{M}_{\text {sat }}-\mathbf{7 2}$ horas } \\
\hline CP & $\mathbf{0 \%}$ & $\mathbf{2 \%}$ & CP & $\mathbf{0 \%}$ & $\mathbf{2 \%}$ & CP & $\mathbf{0 \%}$ & $\mathbf{2 \%}$ \\
\hline CP-A & 461,49 & 492,11 & CP-A & 461,76 & 492,30 & CP-A & 461,63 & 492,34 \\
\hline CP-B & 460,66 & 486,47 & CP-B & 461,03 & 486,41 & CP-B & 460,94 & 486,53 \\
\hline CP-C & 461,74 & 495,95 & CP-C & 461,80 & 496,06 & CP-C & 461,95 & 496,37 \\
\hline MÉDIA & 461,29 & 491,51 & MÉDIA & 461,53 & 491,59 & MÉDIA & 461,50 & 491,74 \\
\hline
\end{tabular}

A massa saturada ficou quase que constante no UHPC e UHPFRC, porém já era esperado que o UHPFRC apresentasse uma massa seca e saturada superior ao UHPC, em consequência da adição de fibras metálicas. A Figura 6 representa a estabilidade da massa saturada ao longo do tempo, algo que já era esperado também, pois na análise microestrutural esse tipo de concreto apresenta baixa permeabilidade e porosidade.

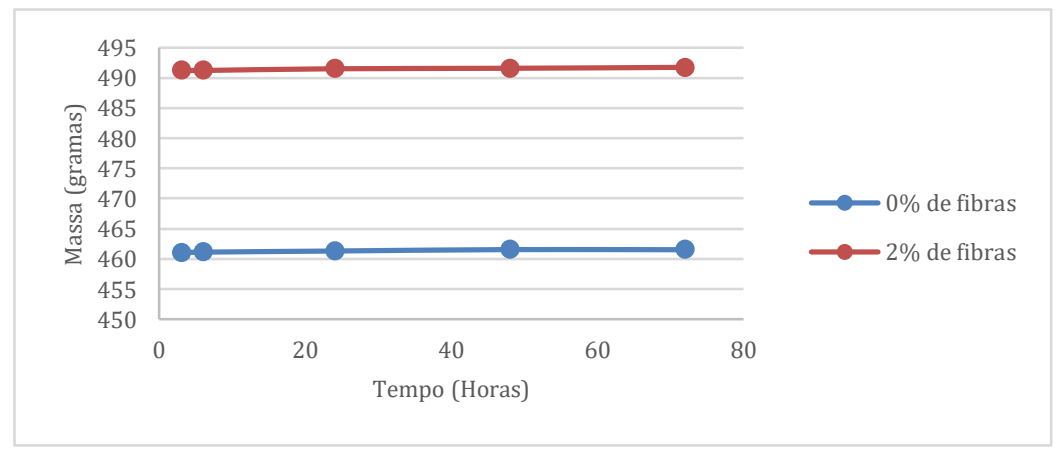

Figura 6 - Determinação da massa saturada.

Como pode ser visto na Figura 7, o UHPC e o UHPFRC apresentam microestrutura quase que impermeável, desse modo pode ser utilizado em processos de reforço, reparo e reabilitação estrutural de estruturas deterioradas ou que apresentam deficiência ou mudança nas solicitações de serviço como, por exemplo, a mudança da sobrecarga.

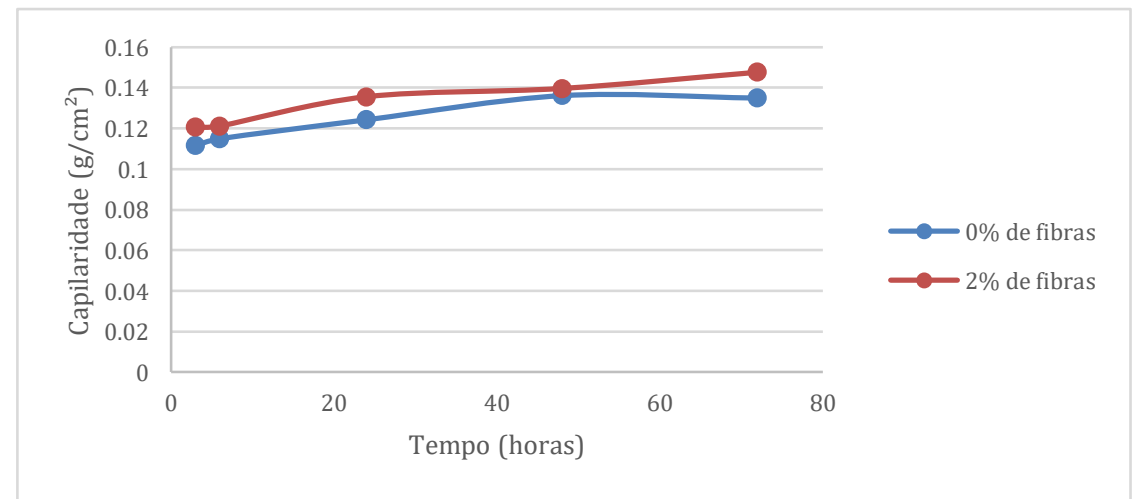

Figura 7 - Determinação da capilaridade.

Durabilidade do UHPC e UHPFRC contendo metacaulim 
Conforme mostra a Figura 7, pode-se afirmar que a absorção por capilaridade do UHPFRC é maior do que no UHPC, cerca de $8 \%$. A adição de fibras aumentou a aborção no UHPFRC, isto pode ser ocorrido por diversos fatores, tais como: possibilidade de um maior índice de vazios, a influencia do formato do $\mathrm{CP}$, fator de forma das fibras e a distribuição das fibras no CP que podem ter prejudicado o adensamento da mistura. Quando se analisa a média apenas da massa saturada (72 horas) com a massa seca do UHPC a variação é de $0,57 \%$. A mesma análise anterior foi feita para o UHPFRC e a variação foi de $0,58 \%$. Como pode ser visto na Figura 8 , foi realizado o ensaio de tração por compressão diametral para medir a altura que a água percolou no corpo de prova, conforme a NBR 7222 (2011). Como pode ser visto, no UHPC não foi constatada a presença de água nos CPs, isso justifica a estabilidade da massa ao longo do tempo. No UHPFRC as fibras impediram a ruptura dos CPs, desse modo não será apresentado. Porém, apresentou estabilidade da massa saturada e baixíssima taxa de absorção por capilaridade.

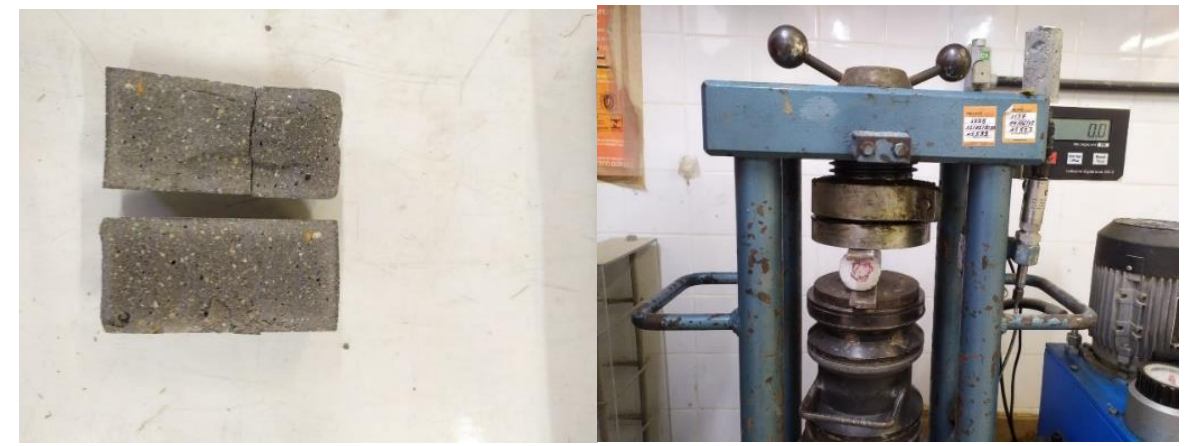

Figura 8 - Ruptura por compressão diametral para análise da altura de percolação da água.

Foi realizada a variação entre a massa saturada do UHPC com $0 \%$ de fibras e do UHPFRC com $2 \%$ de fibras. A variação da massa, como pode ser vista na Figura 9, permaneceu quase que constante variando entre $6,11 \%$ a 6,14\%. Já a variação da capilaridade apresentou maior disparidade de $2 \%$ a $8 \%$. Não foi possível estabelecer uma relação direta entre a variação da massa saturada com a da capilaridade, uma vez que, o teor de fibras foi adicionado na mistura em relação ao volume. Nesse sentido, considerando apenas uma moldagem, o teor de fibras pode variar de CP para CP. Em suma, a capilaridade apresentou um aumento insignificante ao longo do tempo.

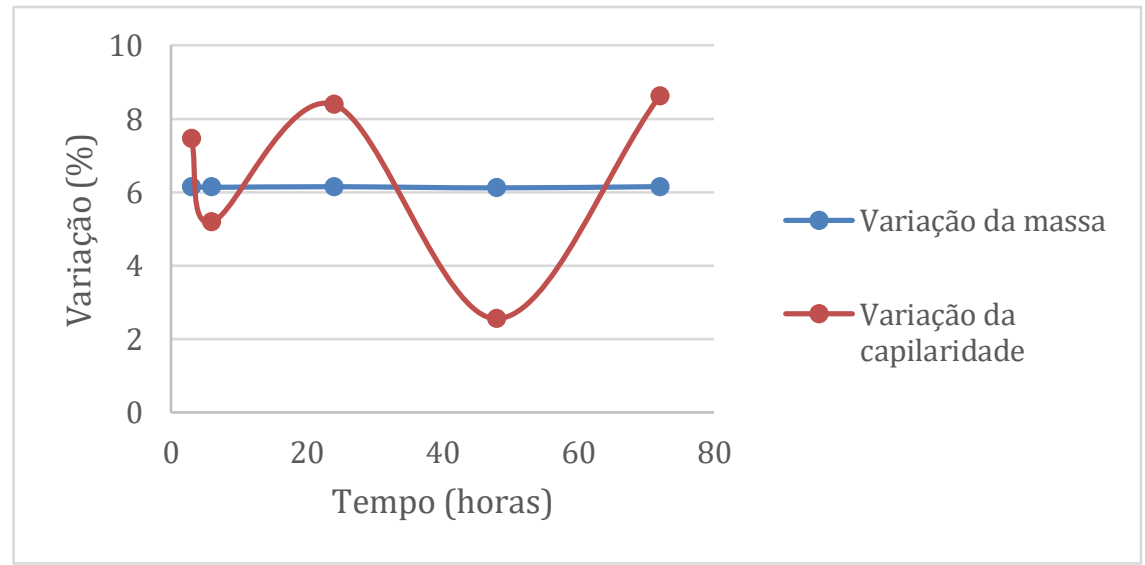

Figura 9 - Variação da massa saturada e da capilaridade.

Em síntese, o comportamento da curva da variação da capiliraridade é senoidal, pois a massa entre os corpos de prova com e sem fibras variam entre si. 


\section{Conclusões}

Percebeu-se que as fibras melhoraram a ductilidade e a resistência à compressão. O UHPC é muito sensível a variação granular, o uso do metacaulim diminuiu o consumo de cimento e reduziu o consumo de água na mistura. A resistência à compressão tende a ser superior em idades elevadas, mas as normas padronizaram a idade de 28 dias, como a hidratação do cimento é lenta e o consumo de cimento é altíssimo nesses concretos especiais, a obtenção da resistência superior a $150 \mathrm{MPa}$ em cura padrão só foi possível pelo altíssimo controle dos materiais, do procedimento de mistura e da cura adequada. Em suma, o UHPC e o UHPFRC apresentaram massa saturada quase que constante com a evolução do tempo, pois a microestrutura do material é quase impermeável, tal característica os torna viáveis em processos de reabilitação estrutural.

Diante dos resultados apresentados, o UHPC e o UHPFRC apresentaram baixa permeabilidade e porosidade, logo os materiais, o traço, procedimento de mistura e o maquinário utilizado na produção desses concretos especiais conseguiram diminuir o índice de vazios de modo que melhorou as propriedades mecânicas e, além disso, dificultou a percolocação da água. Logo, o UHPC e o UHPFRC desenvolvido pode ser utilizado em ambientes que exigem baixa porosidade. As fibras metálicas foram adicionadas, nesse sentido já era esperado uma maior massa no UHPFRC, também percebeu-se que as fibras dificultam a ruptura por compressão diametral para análise da altura de percolação da água. Pelos resultados obtidos, a absorção por capilaridade foi superior no UHPFRC quando comparado ao UHPC.

\section{Referências Bibliográficas}

ALKAYSI, M.; EL-TAWIL, S. Effects of variations in the mix constituents of ultra high performance concrete (UHPC) on cost and performance. Materials and Structures, Springer Science and Business Media LLC, v. 49, n. 10, p. 4185-4200, dez. 2015. Disponível em: <https://doi.org/10.1617/s11527-015-0780-6>.

ALSALMAN, A.; DANG, C. N.; MARTí-VARGAS, J. R.; HALE, W. M. Mixture-proportioning of economical UHPC mixtures. Journal of Building Engineering, Elsevier BV, v. 27, p. 100970, jan. 2020. Disponível em: <https://doi.org/10.1016/j.jobe.2019.100970>.

ASSOCIAÇÃO BRASILEIRA DE NORMAS TÉCNICAS. NBR 9779: Argamassa e concreto endurecidosDeterminação da absorção de água por capilaridade. 2012.

ASSOCIAÇÃO BRASILEIRA DE NORMAS TÉCNICAS. NBR 6118: Projeto de estruturas de concreto - procedimento. Rio de Janeiro, 2014.

ASSOCIAÇÃO BRASILEIRA DE NORMAS TÉCNICAS. NBR 5738: Concreto-procedimento para moldagem e cura de corpos de prova. Rio de Janeiro, 2015.

ASSOCIAÇÃO BRASILEIRA DE NORMAS TÉCNICAS. NBR 7222: Concreto e argamassa-determinação da resistência à tração por compressão diametral de corpos de prova cilíndricos. Rio de Janeiro, 2011.

BAE, Y.; PYO, S. Ultra high performance concrete (UHPC) sleeper: Structural design and performance. Engineering Structures, Elsevier BV, v. 210, p. 110374, maio 2020. Disponível em: <https://doi.org/10.1016/j.engstruct.2020.110374>. 
CAREY, A.; HOWARD, I.; SCOTT, D.; MOSER, R.; SHANNON, J.; KNIZLEY, A. Impact of materials, proportioning, and curing on ultra-high-performance concrete properties. $\mathrm{ACl}$ Materials Journal, v. 117, n. 1, 2020.

CHRIST, R.; PACHECO, F.; SIMONETTI, C.; TUTIKIAN, B. F. Conceitos e aplicações do concreto de ultra alto desempenho: Panorama nacional e internacional. Revista Diálogos: Economia e Sociedade (ISSN: 2594-4320), n. 1, p. 89-102, 2019.

DOIRON, G. Pier repair/retrofit using uhpc-examples of completed projects in north america. In: IOWA STATE UNIVERSITY DIGITAL PRESS. International Interactive Symposium on Ultra-High Performance Concrete. [S.I.], 2016. v. 1, n. 1.

FANG, C.; ALI, M.; XIE, T.; VISINTIN, P.; SHEIKH, A. H. The influence of steel fibre properties on the shrinkage of ultra-high performance fibre reinforced concrete. Construction and Building Materials, Elsevier BV, v. 242, p. 117993, maio 2020. Disponível em: <https://doi.org/10.1016/j.conbuildmat.2019.117993>.

FEHLING, E.; SCHMIDT, M.; WALRAVEN, J.; LEUTBECHER, T.; FRÖHLICH, S. Ultra-high performance concrete UHPC: Fundamentals, design, examples. [S.I.]: John Wiley \& Sons, 2014.

FUNK, J.; DINGER, D. Particle packing. vi: Applications of particle size distribution concepts. Interceram, v. 43, n. 5, p. 350-353, 1994.

HABER, Z. B.; VARGA, I. De la; GRAYBEAL, B. A.; NAKASHOJI, B.; EL-HELOU, R. Properties and behavior of UHPC-class materials. [S.I.], 2018.

MEHTA, P. K.; MONTEIRO, P. J. M. Concrete: Microstructure, Properties, and Materials. [S.I.]: McGraw-Hill Education, 2017.

WALRAVEN, J. The evolution of concrete. Structural Concrete. Lausanne: Fédération Internationale du Béton, V. P1, n. 1, p. 3-12, 1999

$\mathrm{XU}$, L.; WU, F.; CHI, Y.; CHENG, P.; ZENG, Y.; CHEN, Q. Effects of coarse aggregate and steel fibre contents on mechanical properties of high performance concrete. Construction and Building Materials, Elsevier BV, v. 206, p. 97-110, maio 2019. Disponível em: <https://doi.org/10.1016/j.conbuildmat.2019.01.190>. 